\title{
Successful use of a miniaturized bypass system with the DeltaStream extracorporeal rotary blood pump
}

\author{
Stefan Christiansen, MD, ${ }^{\text {a }}$ Christof Göbel, ${ }^{\mathrm{b}}$ Wolfgang Buhre, MD, ${ }^{\mathrm{c}}$ Helmut Reul, $\mathrm{PhD},{ }^{\mathrm{b}}$ and Rüdiger Autschbach, MD, \\ Aachen, Germany
}

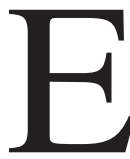
xtracorporeal circulations used for many cardiac procedures might result in an impaired function of nearly all organs. ${ }^{1}$ Beating-heart surgery without extracorporeal circulation is performed more and more to avoid these disadvantages. In this setting revascularization of the right or the circumflex coronary artery might be impossible as a result of severely impaired hemodynamics. ${ }^{2}$ Simplified bypass systems are developed to overcome these problems. ${ }^{3}$ We report on the first successful use of the DeltaStream extracorporeal rotary blood pump (ERBP; Medos AG, Stolberg, Germany) for myocardial revascularization.

\section{Device Description}

The DeltaStream ERBP is developed for versatile extracorporeal circulation. One potential application is intraoperative circulation with a simplified bypass system, which is reduced to its basic components, the pump and the oxygenator. The priming volume of this simplified bypass system is $800 \mathrm{~mL}$ (Figure 1).

The pump has a length of $150 \mathrm{~mm}$, a diameter of $40 \mathrm{~mm}$, a volume of $30 \mathrm{~mL}$, and a weight of $280 \mathrm{~g}$. It has an embedded, long, cylindrical electromotor with an electromechanical efficiency of up to $90 \%$. The motor is surrounded by an annular flow path providing sufficient motor cooling by the blood flow. The DeltaStream ERBP works with moving magnets and stationary coils attached to the housing, and hence there is no air gap between the coils and blood with this motor principle. A thermoelement is fixed onto the motor surface for temperature measurement. The motor and the blood chamber are separated by a polymeric seal. The impeller is positioned between the pump inlet and the motor housing and is supplied with washout holes (Figure 2).

After successful in vitro tests and animal experiments with sheep, the DeltaStream ERBP was approved for clinical use by the Certification Body of the TÜV Product Service (EC Certificate No. G1 0104 15007 017). According to German law, informed consent of the patient is not necessary with this certificate.

\footnotetext{
From the Departments of Cardiothoracic Surgery ${ }^{\mathrm{a}}$ and Anesthesiology ${ }^{\mathrm{c}}$ and the Helmholtz-Institute, ${ }^{\text {b }}$ University of Aachen, Aachen, Germany.

Received for publication March 7, 2002; accepted for publication May 21, 2002 .

Address for reprints: Stefan Christiansen, MD, Department of Cardiothoracic Surgery, University of Aachen, Pauwelsstr 30, 52074 Aachen, Germany (E-mail: schristiansen@ukaachen.de)

J Thorac Cardiovasc Surg 2003;125:43-4

Copyright (C) 2003 by The American Association for Thoracic Surgery

0022-5223/2003\$30.00+0

doi: $10.1067 / \mathrm{mtc} .2003 .122$
}

\section{Clinical Summary}

A 60-year-old male patient was referred for myocardial revascularization because of recurrent angina pectoris. Coronary angiography revealed a high-grade proximal stenosis of the dominant right coronary artery, which was occluded at the crux. Retrograde blood supply was provided by collateral vessels of the left anterior descending coronary artery, which itself did not demonstrate any significant stenoses. The circumflex artery revealed a high-grade stenosis at the bifurcation of the second marginal branch. Left ventricular function was not impaired. Interventional procedures were impossible because of the complex morphologic features of the coronary artery stenoses. Therefore, beating-heart surgery without extracorporeal circulation was planned.

Myocardial revascularization was performed through a complete sternotomy. Tilting of the heart for revascularization of the second marginal branch led to severe hemodynamic impairment, so that the simplified bypass system was instituted with the DeltaStream ERBP through cannulation of the right atrium and the ascending aorta. After unloading of the heart and stabilization of the coronary arteries with the Octopus III device (Medtronic, Düsseldorf, Germany), beating-heart surgery was performed: the left internal thoracic artery was anastomosed with the second marginal branch and the left radial artery with the posterior descending coronary artery. Overall duration of extracorporeal circulation was 85 minutes. Weaning from the simplified bypass system was possible without catecholamine support.

Mechanical ventilation was terminated 4 hours postoperatively. The patient was referred to the normal ward on the first postoperative day. Overall blood loss was $560 \mathrm{~mL}$ without any transfusion requirements. After an uneventful postoperative course, the patient was discharged 7 days later.

\section{Discussion}

Apart from the virtues of new blood pumps, they have to meet the safe and highly accepted standards of conventional blood pumps. Important aspects of blood pumps are lifetime, thrombogenicity, costs, flow characteristics, and blood damage. The major advantages of the DeltaStream ERBP are the small size, the simple design, the low energy requirements (compared with pulsatile blood pumps), avoidance of a venous reservoir, reduced tubing length (by near positioning of the pump to the patient by incorporation of the driving motor into the pump with independence of a driving console), and thus a decreased extracorporeal blood volume. ${ }^{3,4}$ The reduced artificial surfaces and the decreased extracorporeal pressure difference (as a result of the shortened tubes) might result in a reduced blood trauma. Low levels of free plasma hemoglobin were found in animal experiments, ${ }^{3}$ but until now, there is no proof of reduced blood trauma clinically with this device. This potential benefit, as well as the lower filling volume of simplified bypass systems, might lead to reduced transfusion 


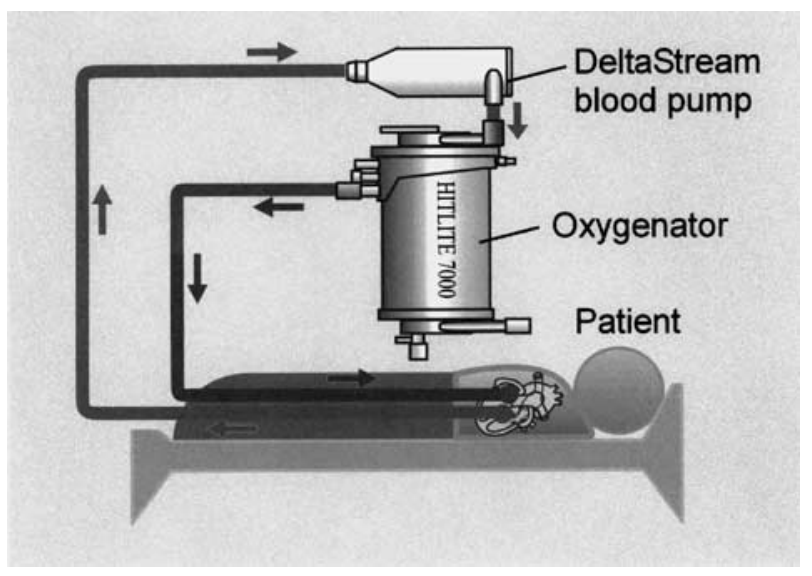

Figure 1. The setting of a simplified bypass system consisting of tubes, an oxygenator, and the DeltaStream ERBP.

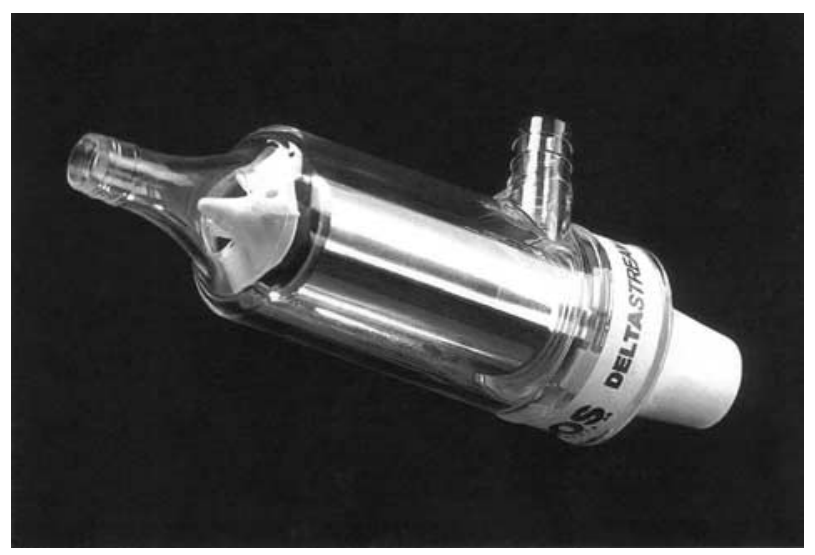

Figure 2. The DeltaStream ERBP.

requirements. Avoidance of low flow areas, which typically appear at the rear sides of the impeller, is necessary to obtain a low thrombogenicity. Therefore, the impeller was supplied with additional washout holes that suck blood from the rear side of the impeller into the main flow region. This setting avoids stagnation of blood flow and secondary thrombus formation on the rear side of the impeller. ${ }^{3}$ By using this impeller design, no thromboembolic events were observed throughout all animal experiments.
The DeltaStream ERBP with an embedded motor might raise the question of thermal loading of blood by means of heat dissipation. Thermal investigations demonstrated an equilibrium with a motor surface temperature of $38.1^{\circ} \mathrm{C}$, which is only $0.6^{\circ} \mathrm{C}$ greater than the reservoir or patient temperature, respectively.

The benefit of simplified bypass systems over conventional full bypass systems remains to be proved, but similar positive results are currently reported by others. Liebold and associates ${ }^{5}$ compared a minimized and closed extracorporeal circuit with a centrifugal pump and a membrane oxygenator as the only active components (the Jostra MECC System) with a conventional cardiopulmonary bypass system. Patients operated with the Jostra MECC System demonstrated significantly lower peak levels of interleukin 6, plasma hemoglobin, troponin T, and S100b. Postoperative blood loss and transfusion requirements were also reduced.

Regarding the clinical standpoint, handling and operation of the DeltaStream ERBP were safe and easy. Hemodynamic instabilities, which sometimes occur in cases of cardiac surgery without extracorporeal circulation during revascularization of back-wall vessels, ${ }^{2}$ were avoided. This facilitates the technical demands of beating-heart surgery and might help to reduce morbidity and mortality by avoiding incomplete revascularization. Furthermore, unloading of the heart with secondary reduced oxygen demands of the myocardium might diminish the risk of intraoperative myocardial infarction because of temporary occlusion of the target vessel.

\section{Conclusion}

Simplified bypass systems, as presented in this case report using the Deltastream ERBP, might be an effective tool to overcome hemodynamic instabilities in beating-heart surgery and might help to reduce its technical demands. Furthermore, impairment of organ function might be reduced, leading to a faster postoperative recovery.

\section{References}

1. Mora CT. Cardiopulmonary bypass. New York: Springer-Verlag; 1995.

2. Autschbach R, Rauch T, Engel M, Brose S, Ullmann C, Diegeler A, et al. A new intracardiac microaxial pump: first results of a multicenter study. Artif Organs. 2001;25:327-30.

3. Göbel C, Arvand A, Eilers R, Marseille O, Bals C, Meyns B, et al. Development of the MEDOS/HIA DeltaStream extracorporeal rotary blood pump. Artif Organs. 2001;25:358-65.

4. Meyns B. Indications for rotary blood pumps in clinical practice. Artif Organs. 2001;25:323-6.

5. Liebold A, Reisinger S, Lehle K, Rupprecht L, Philipp A, Birnbaum DE. Reduced invasiveness of perfusion with a minimized extracorporeal circuit (the Jostra MECC System). Thorac Cardiovasc Surg. 2002;50(Suppl 1):S75. 